Cinémas

Revue d'études cinématographiques

Journal of Film Studies

\title{
Les petites apocalypses de John Cassavetes
}

\section{Jean-François Chassay}

Volume 13, numéro 3, printemps 2003

Imaginaire de la fin

URI : https://id.erudit.org/iderudit/008708ar

DOI : https://doi.org/10.7202/008708ar

Aller au sommaire du numéro

Éditeur(s)

Cinémas

ISSN

1181-6945 (imprimé)

1705-6500 (numérique)

Découvrir la revue

Citer cet article

Chassay, J.-F. (2003). Les petites apocalypses de John Cassavetes. Cinémas, 13(3),

79-94. https://doi.org/10.7202/008708ar

\section{Résumé de l'article}

La sensation du temps est très particulière dans les films de John Cassavetes. Le temps est, dirait-on, étiré, suspendu. Ce « suspense », pour utiliser le terme dans un sens particulier, est souvent celui de l'attente d'une catastrophe à venir, qui se manifeste moins par une action au sens fort que par des signes signalant le basculement des personnages vers une crise, que les mouvements chaotiques de la caméra, tout comme le son, les paroles souvent imprécises, semblent accompagner. Le présent article s'applique à analyser cette apocalypse subjective ou " petite apocalypse " pour reprendre le titre d'un roman de Tadeus Konwicki, à travers Husbands (1970) et The Killing of a Chinese Bookie (1976). 


\section{Les petites apocalypses de John Cassavetes}

\section{Jean-François Chassay}

\section{RÉSUMÉ}

La sensation du temps est très particulière dans les films de John Cassavetes. Le temps est, dirait-on, étiré, suspendu. Ce «suspense", pour utiliser le terme dans un sens particulier, est souvent celui de l'attente d'une catastrophe à venir, qui se manifeste moins par une action au sens fort que par des signes signalant le basculement des personnages vers une crise, que les mouvements chaotiques de la caméra, tout comme le son, les paroles souvent imprécises, semblent accompagner. Le présent article s'applique à analyser cette apocalypse subjective ou "petite apocalypse " pour reprendre le titre d'un roman de Tadeus Konwicki, à travers Husbands (1970) et The Killing of a Chinese Bookie (1976).

\section{ABSTRACT}

The sensation of time is very particular in the films of John Cassavetes. One might say that time is stretched, suspended. This "suspense," to use this term in a special way, is often shaped by the expectation of a catastrophe to come; rather than being manifest by an action in the strong sense of the term, it is signalled by the breakdown of characters on the border of a crisis, by the chaotic movement of the camera, as well as by the sounds and imprecise words that seem to accompany this crisis. This article undertakes an analysis of this subjective apocalypse or "small apocalypse" to use the title of a novel by Tadeus Konwicki, through Husbands (1970) and The Killing of a Chinese Bookie (1976). 
Je pense que personne ne peut vivre sans philosophie.

John Cassavetes

Les rages, les débauches, la folie dont je sais tous les élans et les désastres tout mon fardeau est déposé. Apprécions sans vertige l'étendue de mon innocence.

Arthur Rimbaud

Le romancier et cinéaste polonais Tadeus Konwicki (1981, p. 7) ouvre son roman La Petite Apocalypse de la manière suivante:

Voici venir la fin du monde. Voici ma fin du monde à moi, imminente et sournoise dans sa lente progression de reptile. La fin de mon monde personnel. Mais avant que ce mien univers ne tombe en ruine, avant qu'il ne se désagrège en atomes, il me reste à parcourir le dernier kilomètre de mon Golgotha, [...] monter ou descendre les quelques traverses de l'échelle du non-sens.

On trouve là synthétisé en quelques lignes un mode particulier d'imaginaire de la fin qui résume d'une certaine manière ce qui se vit dans plusieurs films de John Cassavetes.

Cette façon de penser la fin ne repose pas sur le terme du monde: rien ne permet ici d'annoncer la destruction de l'humanité grâce aux pouvoirs technologiques de la science, que ce soit par la vertu de bombes nucléaires ou d'un virus savamment préparé par un scientifique fou (signes d'une fin laïque) et rien ne laisse croire, dans une perspective millénariste par exemple, à une apocalypse assurant le renouvellement cosmique et apportant l'espoir d'une récupération de la béatitude des commencements (versant religieux du phénomène). Il s'agit du terme d'un monde, celui du narrateur, qui voit sa propre fin dans un horizon très proche. Or, cette fin de soi, du sujet, ne peut en toute logique que se penser dans un rapport étroit avec la fin du monde, ce qui se note ici dans la manière dont les deux 
premières phrases s'enchaînent: le roman s'ouvre sur la disparition du monde, apparemment constitutive de la disparition du narrateur qui suit dans l'ordre énonciatif. "Imaginer l'apocalypse est une façon de se venger à l'avance de sa propre mort» (Vidal 1999-2000, p. 45). La fin s'annonce dès le départ et tout le roman se présente en ce sens comme le temps de la fin. Tous les signes qui organisent le texte sont centrés sur le sujet dans ce rapport étroit à sa propre disparition et à l'idée même de disparition.

Je voudrais montrer au cours des prochaines pages comment, dans une perspective non pas littéraire mais cinématographique, John Cassavetes use de la fin justement à travers de petites apocalypses ou des "apocalypses intimes", aussi éloignées qu'on puisse l'imaginer d'une pensée religieuse. Effondrement de soi, mais aussi réflexion sur la faille dans l'imaginaire qu'implique cet effondrement, Cassavetes évite l'aspect spectaculaire et les artifices usuels de sa représentation pour organiser davantage ses films autour d'une pensée de la fin qui repose sur des enjeux esthétiques particuliers. Dans l'essentiel de l'œuvre de Cassavetes (où tragédie et comédie se côtoient), la fin est une pensée, un signe complexe, un système de représentation. Certes, le risque, en interprétant ainsi la fin, consiste à la voir partout au cinéma (la mort n'en est-elle pas un des sujets dominants?). Cependant, la quête de sens, qui produit aussi une crise du sens, se déploie ici à l'intérieur d'un système polymorphe où la pensée de la fin prime, même si elle se révèle souvent de manière implicite. Je m'arrêterai en particulier sur Husbands où la mort est le point de départ de la réflexion (et du film), et sur The Killing of a Chinese Bookie, film sur la mise en scène de la mort et sur une mort qui, en réalité, n’en finit plus.

Après le décès subit de leur ami Stewart Jackson, trois quadragénaires de la bourgeoisie américaine de Long Island vivent pendant quelques jours une cavale adolescente, les conduisant d'un bar de New York où les beuveries sont interminables jusqu'à Londres, où ils iront claquer de l'argent dans un casino, puis draguer des femmes qu'ils entraîneront dans une des chambres. De quatre qu'ils étaient, ils ne sont plus que trois, ce qui brise la symétrie de leurs relations. Sans jamais que le nom 
de Stewart ou les événements relatifs à sa disparition ne soient évoqués (sauf très rapidement à une ou deux reprises et de manière presque anecdotique), Harry (interprété par Ben Gazzara), Archie (Peter Falk) et Gus (John Cassavetes) feront face à la fin d'un monde, à la fin d'une durée, celle de leurs existences bien réglées, entre quatre couples se dirigeant lentement vers la vieillesse. C'est pourquoi ils vivront des choses à la fois banales et ahurissantes pour eux, comme si tout se formulait pour la dernière (ou la première) fois. De la même manière que des paroles insignifiantes peuvent prendre une valeur tragique si elles sont prononcées par un agonisant qui risque de ne plus jamais en répéter de semblables, de même chaque action, chaque geste posé par Harry, Archie et Gus contiennent un poids de véracité et de réalité qui annonce que le monde, pour eux, ne sera plus jamais le même.

Le titre du film a une valeur ironique. Certes, on apprend que les trois hommes vivent une vie bourgeoise assez traditionnelle, qu'à eux trois ils ont huit enfants; de plus, ils évoquent de manière évidente la classe moyenne-supérieure de la côte est américaine. Pourtant, ils échappent tout au long du film à leur statut de maris. Le spectateur n'a pas accès à l'histoire de leur vie, sauf dans une scène où Harry passe chez lui et fait face à sa femme et à sa belle-mère. Or, cette scène laisse justement entendre que mari et femme ne forment plus un couple, l'incommunicabilité entre l'un et l'autre étant patent. La scène ressemble à une parenthèse pénible dans un film qui s'apparente lui-même à une parenthèse, sinon à un rêve, où la mort a aboli la réalité. On pourrait même dire que le film dans son ensemble peut se penser comme une veillée funèbre, une apocalypse au sens premier (du grec apocaluptô), qui signifie révéler, dévoiler, soulever le voile pour découvrir ce qui est caché. Dans la tradition religieuse, on entend alors la révélation d'une entité divine à un être humain, un élu. Mais ici, la révélation se fait dans une éprouvante solitude de l'être, étrangère à toute transcendance. Elle se rapproche plutôt du propos de Levinas (1985, p. 39) pour qui «solitude et matérialité vont ensemble. La solitude n'est pas une inquiétude supérieure qui se révèle à un être quand tous ses besoins sont satisfaits. Elle n'est pas l'expérience 
privilégiée de l'être pour la mort, mais la compagne, si on peut dire, de l'existence quotidienne hantée par la matière». Or, dans le film de Cassavetes, c'est cette quotidienneté revue et corrigée après l'épreuve de la mort qui se signale comme une véritable révélation.

Résumer Husbands en racontant qu'il s'agit de la mort d'un homme, mort qui provoque dans son entourage une crise, ramène le film à bien peu de chose, ce canevas étant comme on le sait relativement commun. L'originalité d'Husbands tient bien sûr à la manière dont Cassavetes filme ses personnages, technique indissociable comme on le sait de son utilisation du temps, qui exprime également une radicale solitude de l'être. Le temps est, dirait-on, étiré, suspendu. Ce «suspense» est souvent celui de l'attente d'une catastrophe à venir, qui se manifeste moins par une action au sens fort que par des signes annonçant le basculement des personnages vers une crise, que les mouvements chaotiques de la caméra, tout comme le son, les paroles souvent imprécises, semblent accompagner. La mort de Stewart est évidemment le signe d'une perte, d'une fin. Mais au-delà de cela, c'est le morcellement du monde qui signale une chute. L'unité est dorénavant impossible et les trois hommes sont confrontés à cette nouvelle réalité. Ce qui disparaît pour eux avec la mort de Stewart, c'est l'univers de la précision, à travers lequel nous vivons depuis près de deux siècles (le progrès, la linéarité du temps technologique qui organise nos vies), au profit du monde de l'à-peu-près; l'investissement toujours plus profond (et parfois douloureux) de notre durée intime à travers le temps mesuré - temps des horloges, temps du travail professionnel, de la routine - éclate. Les signes ne sont plus du même ordre; le travail des uns et des autres ne fonctionne plus, cette réalité de l'organisation de la vie disparaît. Pensons à la scène burlesque où Gus dans son bureau de dentiste tente de calmer une patiente hilare de nervosité, alors qu'il parle à Archie qui tourne autour de lui comme s'il discutait dans son propre salon. Réaliste, la scène est pourtant à côté de la réalité, parce que les signes ne correspondent pas à ce qui est attendu. D'ailleurs, les personnages sont eux-mêmes décalés: contre toute logique, la patiente (qu'on ne reverra plus) est au centre du plan, 
l'assistante dentaire s'occupe de l'essentiel du travail, alors que Gus est penché (on le voit de trois quarts dos) et Archie, en partie hors-champ. Pensons encore à la scène où Harry, dans son bureau, hurle qu'il doit travailler tout en s'empêchant de le faire et en déconcentrant ceux qui l'entourent. Tout au long du film, outre cette désorganisation, la temporalité semble incohérente, étrangement morcelée. Le temps se découpe selon des modalités singulières et on a envie de dire qu'il en est de même pour l'espace: le départ des trois hommes à Londres pour un court laps de temps donne l'impression qu'ils ne font que se déplacer de Long Island à Manhattan, comme si les distances n'avaient aucune importance. Se déroulant sur quelques jours à peine, Husbands donne pourtant peu d'indicateurs temporels. Tout le film, plan après plan, exprime cette désorientation. "I'm so confused", dira à un certain moment un des trois principaux protagonistes, mais ils pourraient tous le répéter à de nombreuses reprises. Ici l'espace et le temps, d'une certaine manière, se confondent: l'espace devient une représentation directe du temps qui échappe à la chronologie. Le "bruit et la fureur", intériorisés par les personnages et exprimés selon différents modes d'expression (mouvements incessants, cris et colères, beuveries, fuite en avant...), ne proposent pas un déroulement, n'offrent pas une direction claire au spectateur, puisque tout est déjà suspendu, arrêté. Les personnages des films de Cassavetes ont beau s'agiter, la situation dans laquelle ils se trouvent pris ne leur offre pas la possibilité de sortir d'une puissante vacuité dont ils sont conscients. La grande force de ses films est de ne jamais expliquer cette vacuité, mais de la laisser voir.

On le sait, la longueur des scènes, le temps passé sur chaque plan, donnent aux films de Cassavetes leur vérité particulière. Pour Emmanuel Levinas (1985, p. 55), «il y a dans la souffrance une absence de tout refuge. Elle est le fait d'être directement exposé à l'être. Elle est faite de l'impossibilité de fuir et de reculer. Toute l'acuité de la souffrance est dans cette impossibilité de recul ». Chez Cassavetes justement, la durée des plans et l'insistance sur les visages rendent impossible ce refuge, elles en sont la marque privilégiée. La proximité des personnages à l'écran est à l'égal de la proximité de la mort. "L'effet de vérité " 
des films de Cassavetes, sur lequel on a tellement glosé, tient sans doute à cette angoisse d'une proximité de la mort, à cette impossibilité de se réfugier hors d'elle. «L'objet que je rencontre est compris et, somme toute, construit par moi, alors que la mort annonce un événement dont le sujet n'est pas le maître, un événement par rapport auquel le sujet n'est plus sujet» (Levinas 1985, p. 57). La durée des scènes a aussi dans Husbands un rapport troublant avec la mort. L'intensité de la durée, de ce temps en suspension, vise pourrait-on dire à "prolonger l'éphémère, sans le perdre" (Carrière 1998, p. 156). La mort de Stewart est une fin à laquelle on s'accroche pour la faire durer, comme si on ne pouvait accepter que le deuil ne dure pas pour l'éternité, que la mort ne se manifeste pas comme un éternel présent, toujours recommencé. Elle apparaît davantage comme un mystère, impalpable, où la solitude de chacun vient s'abîmer.

La scène inaugurale, au cimetière, donne une saisissante impression de cette solitude (de ces solitudes) vécue(s) en contiguïté avec le mystère de la mort, qui ne se démentira pas. Le statut de mari dont il était question plus tôt n'est valable au fond que pour la série de clichés qui ouvrent le film, souvenirs d'une rencontre manifestement bien arrosée entre les quatre couples un été, au bord d'une piscine. Ces photos banales, atemporelles, figées par définition, sont cristallisées comme un souvenir. C'est plutôt la mort qui rend le mouvement possible (de la caméra, de la vie). En répétant plusieurs fois de suite à Archie, alors qu'ils se dirigent vers le cimetière: "Don't believe truth", Gus semble exprimer une sorte de prise de conscience aiguë de l'imperfection scandaleuse du monde. Et cette imperfection tient à l'insupportable passivité du sujet, qui se trouve dans l'impossibilité d'agir. Que peut-on faire devant la mort? Rien d'autre que s'y mirer.

$\mathrm{Au}$ cours de cette première scène, pendant le discours du curé, les trois hommes se frayent chacun un chemin pour se rapprocher du cercueil au sein d'une véritable marée humaine qui se recueille. Prise en plongée, la scène donne l'impression que les trois hommes descendent vers le mort (vers la mort), comme s'il y avait moyen de s'approcher d'elle pour la saisir et, paradoxalement, pour trouver refuge dans ce qui provoque la 
souffrance, cette mort justement insaisissable. J'écris "marée», mais on pourrait dire "forêt» et penser aux premiers vers de $L a$ Divine Comédie de Dante (1985, p. 25) : «Au milieu du chemin de notre vie/je me retrouvai par une forêt obscure/car la voie droite était perdue. " C'est vers un enfer laïcisé, un enfer de l'être que le trio dirige ses pas.

Puis la caméra se rapproche et isole à tour de rôle Archie, Gus et Harry, au milieu de la foule, masques funéraires parmi d'autres ${ }^{1}$. Ils se démarquent néanmoins tous les trois (se sont les seuls à bouger dans cette foule immobile), puis la caméra les isole les uns des autres, avant de les réunir à nouveau dans un plan où on les voit de dos, comme s'ils étaient soudés, alors qu'ils font face au cercueil et à la veuve de Stewart.

Comme l'écrit Raymond Carney (1985, p. 126) : "The gatherings of friends, relatives, and neighbours here and at the preceding pool party are the last crowds we see in Husbands. [...] In the scenes following the funeral scene, Cassavetes pulls his camera back to isolate his three men within large, deserted, and anonymously impersonal visual spaces. " Même à Londres, quand le trio est au casino par exemple, la caméra se focalise sur eux (et sur leur exubérance), masquant largement les clients et les employés qui se trouvent dans les parages. Mais la scène des funérailles a aussi indiqué sémiotiquement que Gus, Archie et Harry sont à la fois seuls ensemble et isolément. Ce paradoxe apparent s'explique justement par la mort qui les isole chacun dans leur souffrance, tout en les rapprochant instinctivement les uns des autres. "Seul un être arrivé à la crispation de sa solitude par la souffrance et à la relation avec la mort, se place sur un terrain où la relation avec l'autre devient possible» (Levinas 1985, p. 64).

Cet isolement se manifeste parfois lors de scènes séparées, parfois au cours de la même scène. Ainsi, dans la scène qui se déroule dans le métro (sorte de caveau funéraire sous terre où la veillée funèbre peut commencer, après le départ du cimetière), les trois hommes sont isolés sans même être situés les uns par rapport aux autres.

Les raccords de regard, soit parce que les personnages ne se regardent pas, soit parce qu'est filmé celui qui regarde celui-qui-écoute-celui-qui-parle (et non celui- 
qui-parle ou celui-qui-écoute-celui-qui-parle) ne fonctionnent pas du tout et ne livrent par conséquent pas au spectateur la géographie de l'espace. Il faudra attendre longtemps le plan large qui les positionne spatialement, Cassavetes ayant préféré d'abord créer un autre espace, celui (à défaut d'un meilleur mot) des caractères (Gavron et Lenoir 1985, p. 123-124).

La distance entre les personnages sera davantage marquée lors de la partie londonienne du film. S'ils sont souvent très proches dans la scène de beuverie new-yorkaise - y compris physiquement: voir notamment le passage qui se déroule dans les toilettes du bar —, s'ils forment encore un groupe homogène lors de leur séjour dans le casino londonien - leur vulgarité détonne - il n'en sera pas toujours de même.

Ainsi, le plan-séquence dans la chambre d'hôtel (les trois hommes avec trois femmes rencontrées par hasard) va rapidement voir le groupe se scinder. Cela se produira après la crise de larmes de Harry, sur laquelle le spectateur n'aura aucune explication. Souvenirs de Stewart qui remontent à la surface? De ce qui s'annonce comme une séparation avec sa femme? Compréhension plus large qu'un monde, son monde a pris fin? Un peu tout cela, sans doute. Peu importe, il en résulte une dispersion et le spectateur verra à tour de rôle chacun des trois hommes tenter d'entrer en relation avec la femme qui l'accompagne. (L'énoncé doit être entendu dans le sens le plus large possible: par exemple, la femme avec laquelle se trouve Archie ne comprend pas l'anglais et n'ouvre pas la bouche pendant toute la scène!)

Chaque scène est très longue, la caméra semble à l'affût du moindre mouvement des acteurs, insistant sur un visage, jusqu’à ce qu'il ait exprimé tout ce qu'il avait à exprimer. Là plus qu'ailleurs on sent ce besoin, pour reprendre l'expression de Jean-Claude Carrière, de vouloir prolonger l'éphémère. Les scènes donnent l'impression que pour Harry, comme pour Gus et pour Archie, il s'agit d'abord de tenir le coup, de maintenir le plus possible le face-à-face avec autrui, de croire à l'avenir, audelà de la mort, dans le cadre d'une relation intersubjective. Mais rien ne fonctionne et chaque scène apparaît comme une 
forme de lente agonie, chaque plan ressemble au constat d'un échec qui épuise la scène des funérailles. La virée à Londres n'aura rien donné, la faille provoquée par la fin de Stewart ne pourra être comblée. La mort est maintenant là, définitivement, tapie dans l'esprit des trois hommes comme une réalité. " $\mathrm{La}$ mort est certaine, cela veut dire qu'elle est toujours possible, possible à chaque instant, mais par là que son "quand" est indéterminé. Tel sera le concept complet de la mort: possibilité la plus propre, possibilité indépassable, esseulante, certaine, indéterminée» (Levinas 1993, p. 63).

Le séjour à Londres se termine très abruptement: une scène se termine qui a lieu dans une chambre, puis Gus et Archie sont dans l'avion et les voilà dans un taxi qui les laisse à proximité de leur maison respective. Lorsqu'ils sortent du taxi, ils se retournent pour regarder la voiture s'éloigner en effectuant l'un et l'autre exactement les mêmes mouvements. Stewart est mort, Harry est resté à Londres. De quatre qu'ils étaient, ils ne sont plus que deux et retrouvent un (précaire) équilibre. Que va devenir Harry demande l'un des deux hommes? Nous ne le savons pas, mais c'est bien le signe que le retour à la normale est tout sauf, justement, normal. Quelque chose est mort en même temps que Stewart. La dernière image en ce sens ne doit pas tromper. Le plan s'arrête, et le film avec lui, quand Gus passe le coin de la maison, sortant du champ dans l'image. Il chute plus qu'il ne retourne en arrière. On ne peut pas vraiment revenir avant la fin.

Les films de Cassavetes, et c'est le cas dans Husbands, offrent souvent des scènes pathétiques ou alors très drôles. Mais il n'existe pas de vérité sociologique chez lui (malgré, par exemple, la critique du racisme qu'on retrouve dans Shadows). On y verra plutôt une vérité ontologique, que Husbands exprime avec beaucoup d'acuité. Voilà qui peut passer inaperçu dans la mesure où il n'y a pas de thèse dans ce film, mais d'abord un rapport émotionnel. Il y a aussi, néanmoins, un rapport intellectuel de l'être au monde dont la manière de filmer de Cassavetes rend compte.

Si Husbands contient des moments sombres, on peut dire que The Killing of a Chinese Bookie est plus précisément un film noir, 
dans la tradition américaine: histoire de meurtres, présence de la pègre auquel un héros solitaire tente d'échapper, action se passant surtout la nuit, etc. Mais comme Gloria avec le film d'action, The Killing of a Chinese Bookie joue avec les codes du genre au point de finir par y échapper.

Cosmo Vitelli (Ben Gazzara) est propriétaire d'une petite boîte de nuit assez minable (un strip joint), le Crazy Horse West, mais qui est la fierté de sa vie et dans laquelle il a investi toutes ses énergies en cherchant à y faire preuve de "classe" et de "style" (deux mots qu'il répète souvent). Dans sa vision des choses, les strip-teaseuses doivent aussi être perçues comme des artistes qui exécutent des numéros (The Gunfight at the O.K. Corral, An Evening in Paris...) sous la férule d'une sorte de Monsieur Loyal appelé Mister Sophistication.

Vitelli effectue le dernier versement qui le libère de la "protection" de la pègre et il décide de fêter l'événement de manière particulière en allant jouer au poker. Mais il perd une somme de 23000 dollars et se trouve de nouveau endetté. En guise de remboursement, la pègre lui propose de tuer un bookmaker chinois qui les gêne. Il commence par refuser, mais on lui fait vite comprendre qu'il n'a pas tellement le choix. Cosmo Vitelli parviendra à effectuer ce meurtre (tout en se faisant blesser) et le gang qui l'a commandé décidera d'éliminer ce témoin gênant. Mais Vitelli réussit à échapper au traquenard qu'on lui a tendu. Il retourne au club et adresse un discours à son personnel, puis au public. On suppose à la fin du film que sa blessure, qui s'est rouverte, va provoquer sa mort, ou que le gang va le rattraper et se débarrasser de lui.

Une fois de plus, le résumé à lui seul ne parvient pas à indiquer ce que produit ici Cassavetes. Film noir, The Killing of a Chinese Bookie n'est pas pour autant un film d'action tel qu'on l'entend habituellement. Malgré son sujet, le film est très lent selon les standards hollywoodiens. L'attention portée aux moindres détails donne à voir énormément d'événements à défaut d'une gamme étendue, linéaire (et prévisible) d'actions. Pourtant, The Killing of a Chinese Bookie est beaucoup moins chaotique et beaucoup plus resserré que Husbands. Dans ce dernier, la mort provoquait le chaos, la fin d'un monde 
déclenchait le désordre, l'univers des protagonistes se disloquait. Dans le cas de The Killing of a Chinese Bookie, au contraire, tous les éléments du drame s'organisent autour de la figure esseulée du propriétaire du club et s'enclenchent selon une logique inéluctable pour permettre la compréhension de son destin, mot qu'il faut considérer comme la clé de ce film.

La première scène, où apparaît en veston blanc kitsch un souriant Cosmo Vitelli, est volontairement trompeuse. Comme la scène suivante qui le voit boire du champagne dans une limousine louée avec trois des danseuses qui font partie du personnel de son club avant d'aller jouer au poker, elle ne sert qu'à masquer la chute prochaine du personnage. Pourquoi décide-t-il de jouer et se laisse-t-il aller à perdre, de plus en plus, au moment même où ses problèmes financiers semblent réglés? Dans un contexte religieux, dont ce film se trouve à des annéeslumière, on dirait que le destin est lié à une faute. Mais quelle faute aurait commise Vitelli ? Aucune, en vérité. C'est un homme banal, mais dont on ne peut nier la dignité, dignité d'autant plus grande qu'il approche d'une mort certaine. Encore plus dramatiquement qu'Archie, Gus et Harry, Cosmo Vitelli est un homme seul. Sans famille - sauf symbolique peut-être, grâce à Betty, une de ses employées dont il est très proche et qui a elle-même une fille, Rachel —, sans vrais amis, sans appartement puisqu'on ne le voit jamais que dans le Crazy Horse West qui apparât comme sa vraie maison, il est plongé dans une solitude qui le confronte à la mort. Plus il se rapproche du mystère de la mort, plus le calme et une sorte d'apaisement semblent l'habiter. «Le destin apparait [...] lorsqu'une vie est considérée comme condamnée, au fond comme une vie qui a d'abord été condamnée, et qui est ensuite devenue coupable» (Benjamin 2000, p. 203).

Un mélodrame aurait raconté la vie de Cosmo Vitelli, justifié ses malheurs (son destin) par un passé malchanceux, etc. Dans The Killing of a Chinese Bookie, le spectateur n'a droit qu'à quelques rares bribes de son passé, et encore, vers la fin alors que tout est déjà joué. Malgré la médiocrité de ce qu'il présente sur scène, cet univers de néons et de clinquant, Vitelli croit profondément en l'art. Refusant le travail bâclé, ne dit-il pas lui- 
même: "Everything takes work"? Maladroit mais franc, aimé de ses employés et employées, c'est un marginal en périphérie des spectacles glamours à grand déploiement que Los Angeles peut proposer par ailleurs (et dont la valeur "artistique» dépend souvent davantage de moyens financiers imposants que d'une pensée et d'un contenu). On pourrait développer longuement à partir de cette remarque la métaphore de l'artiste combattant ceux qui l'empêchent de créer dans une entière liberté, mais ce n'est pas l'objectif de cet article. L'important tient plutôt au simple constat de l'honnêteté foncière de Vitelli, qu'il ne s'agit pas de défendre ou de condamner. L'absence de parti pris clair du film par rapport au personnage permet cependant de mettre davantage en lumière le fait qu'il est pris au piège de son destin, "le destin définissant, en somme, la modalité de ce qui arrive" (Conche 1992, p 50). Vitelli est un personnage auquel on parvient à s'identifier, malgré son individualité non conformiste. En ce sens, on peut le voir correspondre à une métonymie du destin. "Remarquons en passant qu'en français une personne, un certain homme, c'est aussi bien "personne", aucun homme: écho du lien original qui soude le déterminé au non-déterminé, le quelque chose au n'importe quoi, la présence de mille chemins à l'absence de tout chemin" (Rosset 1977, p. 16-17). Au cœur de ces chemins, de cette forêt obscure, Vitelli devient une clé permettant de conduire à l'inéluctabilité.

Le meurtre du bookmaker chinois va réussir, dirait-on, miraculeusement. En effet, Vitelli a réussi, après avoir neutralisé les chiens, à échapper aux gardes du corps (il en abat d'ailleurs deux) et à retourner sans encombre au club après avoir effectué un détour par la maison de Betty. Irréaliste, pensera-t-on. Comment un tueur amateur - même si on prend la peine de nous faire savoir qu'il a participé à la guerre de Corée et qu'il a tué - peut-il ainsi s'en tirer comme un tueur à gages aguerri ? Cet éventuel scepticisme du spectateur, sans doute recevable selon une certaine lecture du film, ne prend pas en compte justement le poids du destin qui conduit Vitelli vers la mort (celle du Chinois et, par effet d'enchaînement, la sienne). Les membres de la pègre qui l'entourent dans la voiture lui expliquent de manière extrêmement détaillée ce qu'il doit faire et ce 
qui va se passer. Véritable moira - ils sont d'ailleurs trois à l'entourer, une «mythologie» de la pègre ayant remplacé celle des divinités grecques - , ces hommes scellent son destin en l'envoyant effectuer le meurtre ${ }^{2}$. Ce faisant, ils plongent Vitelli dans une véritable double contrainte: pour échapper à son destin (la faute, c'est-à-dire sa dette de 23000 dollars), il doit pourtant suivre son destin (tuer, et conséquemment commettre une faute beaucoup plus grave). À partir du moment où la faute a lieu, il ne peut que s'enferrer, tous les signes de la mort s'organisant autour de lui, accentuant sa précarité fondamentale. Les marques de l'angoisse dans le visage de Vitelli se multiplient, donnant une tonalité affective fondamentale à son corps à mesure qu'il sent que la fin approche. L'apaisement qu'il paraît ressentir dans les dernières scènes du film donnent l'impression qu’il se situe déjà au-delà de la mort.

Le destin ne se laisse entièrement saisir qu'à travers des signes, non en lui-même, car, s'il se peut que tel trait du caractère, tel enchaînement du destin s'offrent immédiatement au regard, l'ensemble cohérent que visent ces concepts n'est jamais disponible autrement que dans des signes, parce qu'il se situe au-delà de ce qui peut se voir immédiatement (Benjamin 2000, p. 199).

La force du film de Cassavetes tient justement au double registre qui consiste, d'une part, à rendre extrêmement crédible et réaliste la situation du pauvre type pris dans les rets de la pègre, et, d'autre part, à multiplier les signes qui ne cessent de prédire ce que les scènes réalistes du film ne disent jamais directement. Pendant la partie de poker par exemple, le regard de Rachel, le mouvement de jambes d'une autre de ses employées, qui trahit sa nervosité, annonce la catastrophe à venir. Contrairement à la plupart des films de Cassavetes, et notamment à Husbands, The Killing of a Chinese Bookie est peu bavard. Cependant, les agissements des gangsters, tout en silence feutré et marqués par des gestes ou des regards extrêmement significatifs, annoncent sans cesse ce qui attend Vitelli.

The gangsters he must deal with would regard a movie such as The Godfather as a sentimental period piece. 
For all of its bloodthirstiness, Coppola's mafia functions with the codes of honor and personal loyalty of an extended Italian family. [...] The mobsters who call Cosmo into a back office at the casino and require him to sign a "Form 223" and a "Form 17" perform less with the gusto of blood brothers, than the mechanical efficiency and impersonality of the Small Business Loan Department at Chase Manhattan or a cold-blooded group of venture capitalists assessing an investment opportunity (Carney 1985, p. 233).

"Suspense" écrivais-je plutôt: l'action est suspendue, comme la mort elle-même. On ne voit pas Cosmo Vitelli mourir, mais la mort se trouve pourtant là, tapie dans la nuit qui entoure son club lorsqu'il sort dehors, la dernière fois qu'on le voit à l'écran. Rien de plus poignant que cette dernière scène, sans effet particulier, où il essuie négligemment sur son veston le sang qui coule de sa blessure rouverte alors qu'il regarde au loin dans la nuit. Il vit toujours, mais le sang est le signe de la mort sur son corps, signe qu'il refuse de voir, préférant ouvrir les yeux sur la nuit, vers une fin indistincte, mais toute proche, qu'on ne verra pas. Cette nuit, de manière un peu facilement métaphorique, on pourrait dire évidemment que c'est également la mort. De manière plus subtile - et plus marquée en ce sens dans The Killing of a Chinese Bookie que dans Husbands -, on pourrait avancer que l'œuvre de Cassavetes répond à une double contrainte exprimée par de nombreux philosophes sur la mort. Pour paraphraser Kant, on pourrait dire qu'on ne connaît pas la mort. Si je suis en mesure d'en parler, c'est que je ne la connais pas; si je la connais, je ne peux en parler. Ces deux films, d'une certaine manière, nous conduisent dans la mort, dans un temps qui n'existe pas, hors de sa représentation cinématographique.

La fin chez Cassavetes est rarement spectaculaire. Dans Husbands, on pourrait avancer qu'elle se produit avant, et déclenche le chaos du film; dans The Killing of a Chinese Bookie elle est attendue après, et tout semble y mener. L'imaginaire de la fin chez Cassavetes conduit à en traquer les signes dans le visage, le corps, les gestes et les pensées des personnages. Le réalisateur prend le temps d'exposer les effets de la fin chez les êtres qu'il met en scène. Cela coïncide avec un sentiment 
d'étonnement chez eux. La part de tragique chez Cassavetes, un tragique américain, comme on ne le retrouve chez aucun autre réalisateur des États-Unis, tient pour beaucoup dans la découverte progressive de la finitude qui vient frapper de plein fouet ses personnages. Pour résumer son œuvre, Cassavetes aurait pu reprendre une formule de Sophocle (Antigone, cité par Rosset 1977, p. 14) : "Il y a beaucoup de choses étonnantes, mais la plus étonnante de toutes, c'est l'homme.»

\section{Université du Québec à Montréal}

\section{NOTES}

1. Au milieu du film, lorsqu'ils débarquent à Londres, il est frappant de constater qu'au milieu des visages souriants des employés, à l'aéroport ou à l'hôtel, ils retrouvent ce masque funéraire.

2. En ce sens, rien de plus ironique que d'entendre un mafioso, au moment où Vitelli signe ses reconnaissances de dette s'écrier: "There's hope, there's hope, there's hope."

\section{RÉFÉRENCES BIBLIOGRAPHIQUES}

Benjamin 2000 : Walter Benjamin, «Destin et caractère», Euvres I, Paris, Gallimard, 2000.

Carney 1985 : Raymond Carney, American Dreaming, Berkeley, University of California Press, 1985.

Carrière 1998: Jean-Claude Carrière, "Les questions du Sphinx", dans Jean-Claude Carrière (et al.), Entretiens sur la fin des temps, Paris, Fayard, 1998, p. 137-234.

Conche 1992 : Marcel Conche, Temps et destin, Paris, PUF, 1992.

Dante 1985: Dante, L'Enfer, Paris, Flammarion, 1985.

Gavron et Lenoir 1985: Laurence Gavron et Denis Lenoir, John Cassavetes, Paris, Rivages, 1985.

Levinas 1985: Emmanuel Levinas, Le Temps et l'autre, Paris, PUF, 1985.

Levinas 1993 : Emmanuel Levinas, Dieu, la Mort et le Temps, Paris, Grasset, 1993.

Rosset 1977 : Clément Rosset, Le Réel. Traité de lidiotie, Paris, Minuit, 1977.

Vidal 1999-2000: Jean-Pierre Vidal, "Moi seule en être cause...", Protée, vol. 27, $\mathrm{n}^{\circ} 3,1999-2000$, p. 45-56. 